\title{
锆基路易斯酸催化 $\gamma$-戊内酯与胺的反应研究
}

\author{
孔庆山 ${ }^{a, b}$ 李兴龙 ${ }^{b}$ 许华建*,a 傅 尧*,b \\ ( ${ }^{a}$ 合肥工业大学食品与生物工程学院 合肥 230601) \\ $\left({ }^{b}\right.$ 中国科学技术大学化学系 合肥微尺度物质科学国家实验室 能源材料化学协同创新中心 \\ 中国科学院城市污染物转化重点实验室 安徽省生物质洁净能源重点实验室 合肥 230026)
}

\begin{abstract}
摘要 $\gamma$-戊内酯(GVL)是重要的生物质平台分子, 将其转化为高附加值的化学品和燃料, 具有重要的应用前景. 介绍了 一种在温和条件下通过还原胺化/环化反应，分别使用锆基路易斯酸催化剂 $\mathrm{Zr}-\mathrm{P}-\mathrm{O}$ 和 $\mathrm{ZrOCl}_{2} \cdot 8 \mathrm{H}_{2} \mathrm{O}$, 催化 GVL 与胺类 化合物合成差基酰胺类化合物和吡咯烷酮类化合物. 特别值得一提的是，在无溶剂的情况下，均可以得到中等偏上的 产物收率. 该方法为 GVL 的应用研究进一步奠定了基础.
\end{abstract}

关键词 $\gamma$-戊内酯; 羊基酰胺; 吡咯烷酮; 路易斯酸催化; 锆基催化剂

\section{Study on Reaction of $\gamma$-Valerolactone and Amine Catalyzed by Zirconium-Based Lewis Acids}

Kong, Qingshan ${ }^{a, b} \quad \mathrm{Li}$, Xinglong $^{b} \quad \mathrm{Xu}$, Huajian $^{*, a} \quad \mathrm{Fu}, \mathrm{Yao}^{*, b}$

$\left({ }^{a}\right.$ School of Food and Biological Engineering, University of Science and Technology of China, Hefei 230601)

( ${ }^{b}$ Hefei National Laboratory for Physical Sciences at the Microscale, Collaborative Innovation Center of Chemistry for Energy Materials, Chinese Academy of Sciences Key Laboratory of Urban Pollutant Conversion, Anhui Province Key Laboratory of Biomass Clean Energy, Department of Chemistry, University of Science and Technology of China, Hefei 230026)

\begin{abstract}
Valerolactone (GVL) is an important biomass platform molecule, it can be converted into high value-added chemicals and fuel, which has important application prospects. This article describes a method for the synthesis of hydroxyamides and pyrrolidones from GVL and amine compounds by reductive amination/cyclization reactions under mild conditions using zirconium-based Lewis acid catalysts $\mathrm{Zr}-\mathrm{P}-\mathrm{O}$ and $\mathrm{ZrOCl}_{2} \bullet 8 \mathrm{H}_{2} \mathrm{O}$, respectively. In particular, a moderately high product yield can be obtained with the absence of a solvent. This method further lays the foundation for the application research of GVL.

Keywords $\gamma$-valerolactone; hydroxyamide; pyrrolidone; Lewis acid catalysis; zirconium-based catalyst
\end{abstract}

化石资源的消耗带来严重的环境问题及能源危机， 开发基于生物质资源的转化途径来制备燃料和化学品, 进而替代石油资源原料受到了广泛关注. 生物质三组分 纤维素、半纤维素及木质素可以转化为多种重要的生物 质平台分子及其衍生物, 如乙酰丙酸(LA)、 $\gamma$-戊内酯 (GVL)、5-羟甲基糠醛(HMF)、2,5-呋喃二甲酸(FDCA) 等 ${ }^{[1]}$. 转化平台分子及其衍生物制备高附加值的化学品
和燃料成为开发利用生物质资源的重要途径。

GVL 作为重要的平台分子之一, 可以从纤维素或 者半纤维素通过水解或者醇解得到的乙酰丙酸或乙酰 丙酸酯加氢得到. GVL 具有多种重要的用途, 可用作树 脂溶剂、润滑剂、增塑剂、胶凝剂、汽油添加剂、食用 香料及药物中间体等. GVL 可以通过多种反应转化为多 种高附加值化合物，包括燃料添加剂 2-甲基四氢呋

\footnotetext{
* Corresponding authors. E-mail: hjxu@hfut.edu.cn; fuyao@ustc.edu.cn

Received March 8, 2020; revised April 2, 2020; published online April 17, 2020.

Project supported by the National Key Research and Development Program of China (No. 2018YFB1501604), the National Natural Science Foundation of China (No. 21472033), the Key Research and Development Program Projects in Anhui Province (No. 201904a07020069), and the Fundamental Research Funds for the Central Universities.

国家重点研发项目(No. 2018YFB1501604)、国家自然科学基金(No. 21472033)、安徽省重点科研开发项目(No. 201904a07020069)、中央高校基础研究 基金资助项目
} 


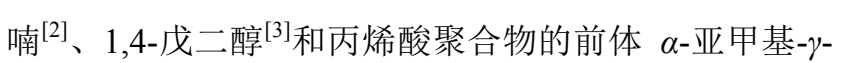
戊内酯 ${ }^{[4]}$ 、香料戊酸酯 ${ }^{[5]}$ 、燃料前体壬酮 ${ }^{[6]}$ 、尼龙 66 前 体己二酸 ${ }^{[7]}$ 等(Scheme 1). 因此开发更多基于 GVL 的高 附加值下游产品, 对于拓宽生物质产业链整体附加值具 有重要意义.

羟基酰胺可作为新型的表面活性剂 ${ }^{[8]}$ 、金属螯合 剂 $^{[9]}$ 、保护基团 ${ }^{[10]}$ 以及重要的药物中间体 ${ }^{[11]}$. 传统制备 羟基酰胺主要使用丁内酯或者 GVL 为原料与胺类化合 物反应制备 ${ }^{[12]}$. 相比于较易反应的丁内酯, GVL 的反应 活性较弱, 需要在高温高压(900 MPa)下反应较长时间 才可以得到较满意的收率 ${ }^{[13]}$. 使用 $\mathrm{AlCl}_{3}$ 作为催化剂可 以显著降低反应条件, 得到较高的产物收率 ${ }^{[14]}$, 这说明 Lewis 酸催化剂的添加对从 GVL 获得羟基酰胺有明显 的促进作用. 但在更加温和的条件下, 以 GVL为内酯原 料得到高选择性的着基酰胺产物仍然具有较大挑战.

吡咯烷酮可以作为溶剂、表面活性剂、药物的关键 中间体, 也是多种清洁剂、墨水和气溶胶重要的成 分 ${ }^{[15]}$. 常规的制备方法主要是使用 LA 与胺类化合物进 行还原胺化反应制备, 已经探索了各种金属催化剂[包 括负载型金属催化剂(即 $\mathrm{Pt}, \mathrm{Pd}, \mathrm{Rh}, \mathrm{Ru}, \mathrm{Co}, \mathrm{Ni}$ )与均相金 属( $\mathrm{Ir}, \mathrm{Ru}$ 的络合物)]以及无金属催化剂 ${ }^{[16]}$. 以 LA 为原 料的还原反应通常需要使用甲酸、有机硅烷或者氢气作 为氢源. 其中, 甲酸具有腐蚀性, 有机硅烷的价格高昂, 高压氢气具有一定的操作危险性. 因此, 迫切需要开发 更加安全、廉价和环保的反应体系, 实现吡咯烷酮类化 合物的高效制备.

$\mathrm{ZrOCl}_{2} \cdot 8 \mathrm{H}_{2} \mathrm{O}$ 作为一种廉价高效的路易斯催化剂, 近年来在有机合成中被广泛使用 ${ }^{[17]}$. 例如催化酚类化 合物的硝化反应 ${ }^{[18]}$, 醇、酚、胺和硫醇的酰化反应 ${ }^{[19]}$, 羧
酸和醇的酯化反应 ${ }^{[20]}$, 胺、吲哚与 $\alpha, \beta$-不饱和酮的迈克 尔加成反应 ${ }^{[21]}$, 醛、苯胺和酮的曼尼希反应 ${ }^{[22]}$, 醛和亚 胺的烯丙基化反应 ${ }^{[25]}$. $\mathrm{ZrOCl} \cdot 8 \mathrm{H}_{2} \mathrm{O}$ 也可以催化合成 $\beta$ 烯胺酮、 $\beta$-烯胺酯 ${ }^{[23] 、} \alpha$-氨基膦酸酯 ${ }^{[24]}$. 此外, 以 $\mathrm{ZrOCl}_{2} \bullet 8 \mathrm{H}_{2} \mathrm{O}$ 为原料合成的非均相催化剂磷酸锆 $\mathrm{Zr}-\mathrm{P}-\mathrm{O}$, 作为一种高效的固体酸催化剂, 也被用于多种 反应中. Cavani 等 ${ }^{[26]}$ 报道了非均相催化剂 Zr-P-O 能够催 化纤维素高选择性地水解制备葡萄糖，结果表明其是一 种高活性的固体酸催化剂. Zr-P-O 催化剂还可以将纤维 素转化为 $\mathrm{C} 6$ 醛糖醇 ${ }^{[27]}$, 催化 LA 加氢制备 $\mathrm{GVL}^{[28]}$, 微 波加热条件下催化果糖和菊粉生产 $\mathrm{HMF}^{[29]}$.

根据文献报道, $\mathrm{Zr}-\mathrm{P}-\mathrm{O}$ 与 $\mathrm{ZrOCl}_{2} \bullet 8 \mathrm{H}_{2} \mathrm{O}$ 都是高效的 Lewis 酸催化剂, 但 $\mathrm{Zr}-\mathrm{P}-\mathrm{O}$ 的酸性相比于 $\mathrm{ZrOCl}_{2} \bullet 8 \mathrm{H}_{2} \mathrm{O}$ 有一定程度的下降. 同时 Lewis 酸催化剂对羟基酰胺的 制备有明显的促进作用，且羟基酰胺经过酸催化环合， 可以进一步得到吡咯烷酮类化合物, 这两个反应都需要 Lewis 酸催化剂的参与 ${ }^{[30]}$.

因此, 我们尝试使用 $\mathrm{ZrOCl}_{2} \cdot 8 \mathrm{H}_{2} \mathrm{O}$ 以及 $\mathrm{Zr}-\mathrm{P}-\mathrm{O}$ 作为 催化剂, 以 GVL 与苯胺作为模型底物, 考察催化剂在反 应过程中的催化效果, 研究反应条件及底物类型对产物 收率的影响，同时对反应机理进行探究.

\section{1 结果与讨论}

首先, 研究了不同的酸催化剂对反应的影响(表 1). 使用固体酸催化剂 Amberlyst-15 时, 反应效果比较差, 检测到产物 4-差基-N-苯基戊酰胺(1)的收率仅为 3\%，5甲基-1-苯基-2-吡咯烷酮(2)的收率为 15\%(表 1, Entry 1). 当使用 $\mathrm{ZrOCl}_{2} \cdot 8 \mathrm{H}_{2} \mathrm{O}$ 作为催化剂时, 产物 2 的选择

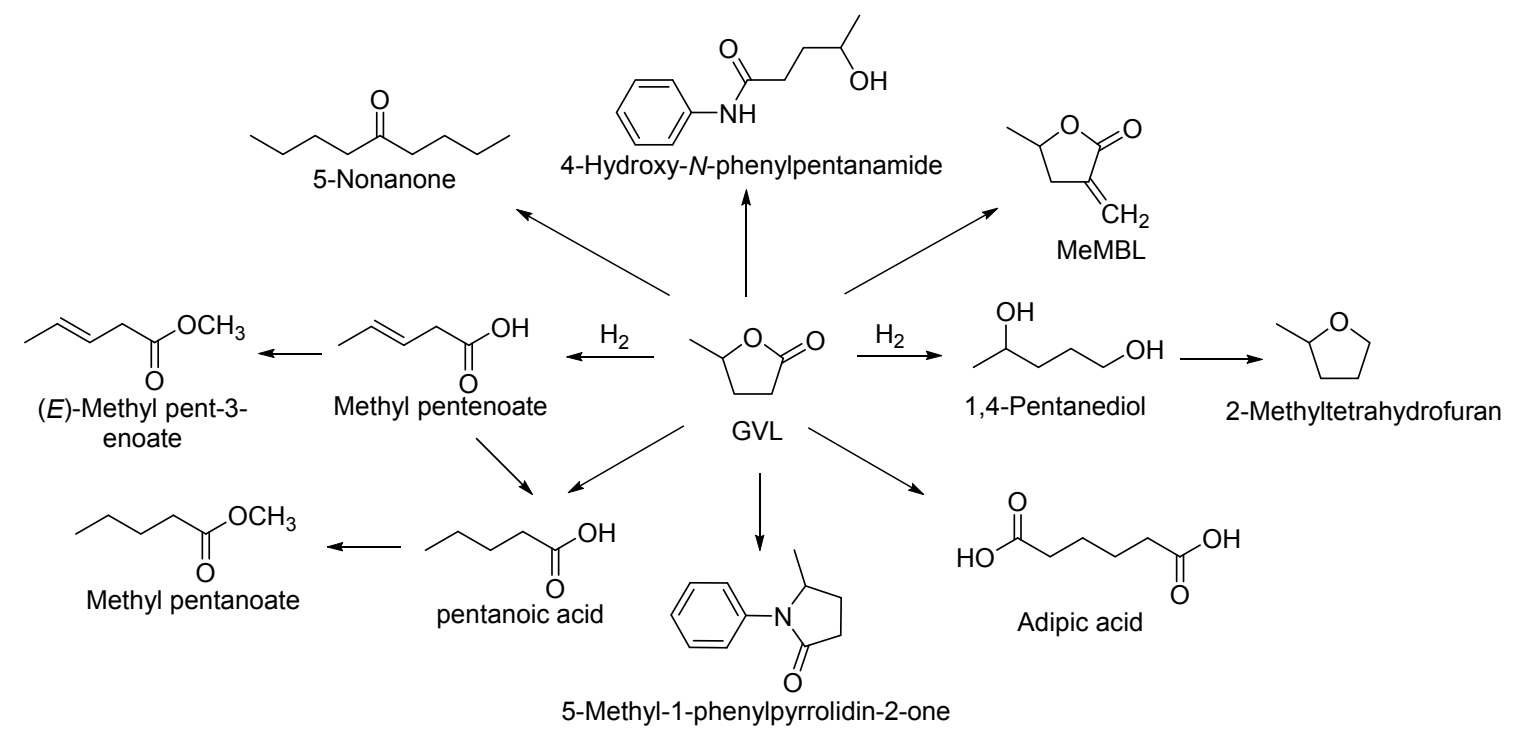

图式 1 GVL 转化为高附加值化学品的反应途径

Scheme 1 Reactions pathways for the conversion of GVL into high value-added chemicals 
性与产率有了明显的提升，在 $100{ }^{\circ} \mathrm{C}$ 下反应 $24 \mathrm{~h}$ 后，产 物 2 的收率为 $52 \%$ ，产物 $\mathbf{1}$ 的收率仅有 $2 \%$ (表 1, Entry 2). 这可能是由于 $\mathrm{ZrOCl}_{2} \bullet 8 \mathrm{H}_{2} \mathrm{O}$ 酸性较强, 使羰基碳更容易 受到胺的亲核进攻, 主要得到环化产物, 从而提高了产 物的收率和选择性 ${ }^{[17 b]}$. 继续篮选其它的 Lewis 酸催化 剂 $\mathrm{SnCl}_{4} 、 \mathrm{AlCl}_{3}$ (表 1, Entries 3 和 4), 发现均有预期的产 物 1 和 2 生成, 但是选择性和收率均比较低, 相比之下, $\mathrm{ZrOCl}_{2} \cdot 8 \mathrm{H}_{2} \mathrm{O}$ 的催化效果最好. 基于此, 进一步笁选了 其他的锆催化剂, $\mathrm{Zr}(\mathrm{OH})_{4}, \mathrm{ZrSiO}_{4}, \mathrm{Zr}-\mathrm{P}-\mathrm{O}$ (表 1, Entries 5 7), 其中, $\mathrm{Zr}(\mathrm{OH})_{4}$ 与 $\mathrm{ZrSiO}_{4}$ 的催化活性较低, 这可 能是由于这两种锆催化剂的酸性较弱. 而非均相催化剂 $\mathrm{Zr}-\mathrm{P}-\mathrm{O}$ 的反应液中产物 $\mathbf{2}$ 的含量很少 $(2 \%)$, 但是产物 $\mathbf{1}$ 的收率达到了 $46 \%$, 表现出了对产物 1 的高选择性. 接 着, 分别对 $\mathrm{ZrOCl}_{2} \bullet 8 \mathrm{H}_{2} \mathrm{O}$ 与 $\mathrm{Zr}-\mathrm{P}-\mathrm{O}$ 两种催化体系进行了 条件的优化(表 1, Entries 9 13). 升高温度到 $120{ }^{\circ} \mathrm{C}$, 延长时间到 $48 \mathrm{~h}, \mathrm{ZrOCl}_{2} \bullet 8 \mathrm{H}_{2} \mathrm{O}$ 能够催化苯胺与 $\mathrm{GVL}$ 反 应, 得到收率为 $63 \%$ 的产物 2 , 对于 $\mathrm{Zr}-\mathrm{P}-\mathrm{O}$ 催化剂, 同 样能够以 $57 \%$ 的收率得到产物 1 .

表 1 反应条件的篮选 ${ }^{a}$

Table 1 Screening of reaction conditions

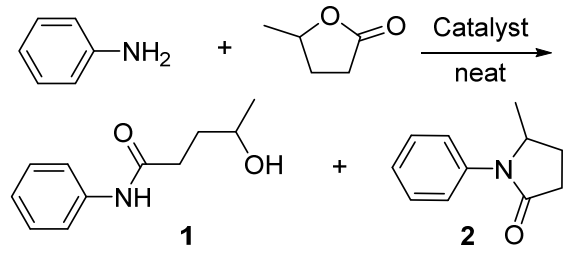

\begin{tabular}{|c|c|c|c|c|c|}
\hline Entry & Catalyst & $T /{ }^{\circ} \mathrm{C}$ & $t / \mathrm{h}$ & Yield $/ \%$ of 1 & Yield $/ \%$ of 2 \\
\hline 1 & Amberlyst- 15 & 100 & 24 & 3 & 15 \\
\hline 2 & $\mathrm{ZrOCl}_{2} \bullet 8 \mathrm{H}_{2} \mathrm{O}$ & 100 & 24 & 5 & 52 \\
\hline 3 & $\mathrm{SnCl}_{4}$ & 100 & 24 & 28 & 23 \\
\hline 4 & $\mathrm{AlCl}_{3}$ & 100 & 24 & 34 & 21 \\
\hline 5 & $\mathrm{Zr}(\mathrm{OH})_{4}$ & 100 & 24 & $<1$ & $<1$ \\
\hline 6 & $\mathrm{ZrSiO}_{4}$ & 100 & 24 & 21 & 13 \\
\hline 7 & $\mathrm{Zr}-\mathrm{P}-\mathrm{O}$ & 100 & 24 & 46 & 2 \\
\hline 8 & $\mathrm{ZrOCl}_{2} \bullet 8 \mathrm{H}_{2} \mathrm{O}$ & 80 & 24 & 32 & 14 \\
\hline 9 & $\mathrm{ZrOCl}_{2} \cdot 8 \mathrm{H}_{2} \mathrm{O}$ & 120 & 24 & 0 & 58 \\
\hline 10 & $\mathrm{ZrOCl}_{2} \cdot 8 \mathrm{H}_{2} \mathrm{O}$ & 120 & 48 & $\mathbf{0}$ & 63 \\
\hline 11 & Zr-P-O & 80 & 24 & 28 & $<1$ \\
\hline 12 & Zr-P-O & 120 & 24 & 49 & 3 \\
\hline 13 & Zr-P-O & 120 & 48 & 57 & 4 \\
\hline
\end{tabular}

${ }^{a}$ Reaction conditions: GVL (1 mmol), aniline (1.1 mmol), catalyst (Entry 1, 50 mg; Entries 2 6 and 8 9, $0.15 \mathrm{mmol}$; Zr-P-O, $100 \mathrm{mg}$ ).

发现在无溶剂体系下, 使用 $\mathrm{ZrOCl}_{2} \cdot 8 \mathrm{H}_{2} \mathrm{O}$ 与 $\mathrm{Zr}-\mathrm{P}-\mathrm{O}$ 催化剂可以分别得到较高的产物选择性. 紧接着对溶剂 的影响进行了考察(图 1). 实验表明, 在两种催化体系 下，以二氯甲烷、二甲基亚砜、四氢呋喃、甲苯以及水 作为溶剂 $(1 \mathrm{~mL})$, 产物 $\mathbf{1}$ 与 $\mathbf{2}$ 的收率均有不同程度的下 降. 在不改变其他条件的情况下, 使用乙腈作为溶剂, $\mathrm{Zr}-\mathrm{P}-\mathrm{O}$ 作为催化剂时, 产物 1 的收率为 $62 \%, \mathrm{ZrOCl}_{2}$ •
$8 \mathrm{H}_{2} \mathrm{O}$ 作为催化剂时检测到产物 2 的收率为 $71 \%$. 接下 来, 采用乙腈作为溶剂的最优反应条件进行了底物的拓 展研究.
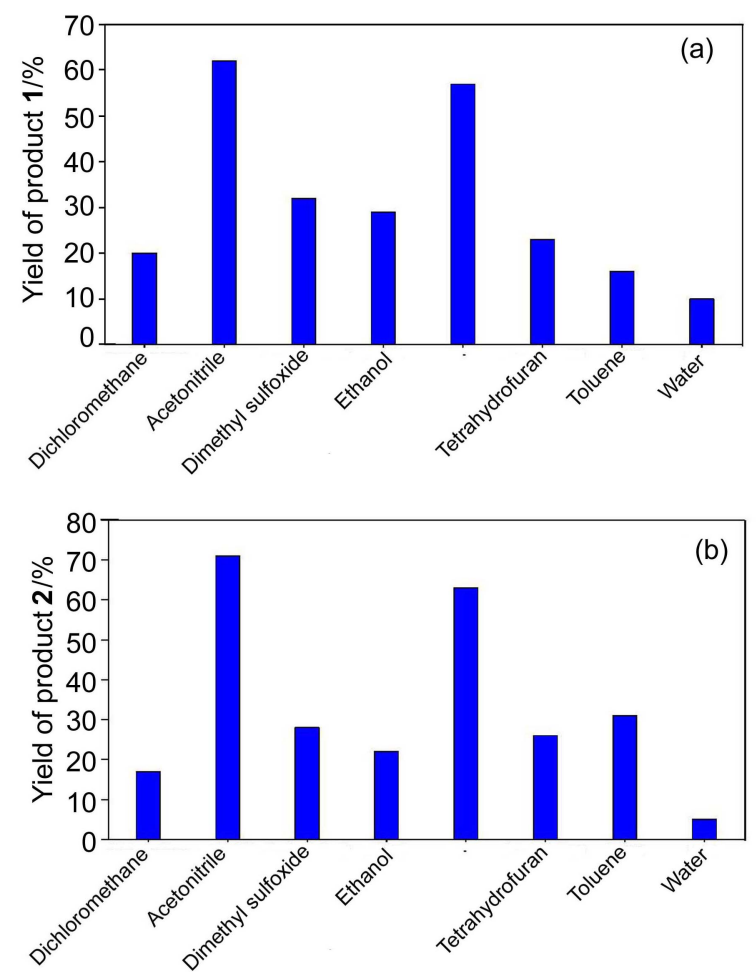

图 1 溶剂的䇥选

Figure 1 Screening of solvents

(a) Product 1; (b) product 2. Reaction conditions: GVL (1 mmol), aniline (1.1 mmol), catalyst: (a) Zr-P-O, $100 \mathrm{mg}$; (b) $\mathrm{ZrOCl}_{2} \bullet 8 \mathrm{H}_{2} \mathrm{O}, 50 \mathrm{mg}$, solvent $(1 \mathrm{~mL}), 120{ }^{\circ} \mathrm{C}, 48 \mathrm{~h}$

更换不同的胺和内酯，进一步来考察反应的适用范 围(表 2). 使用该方法，反应底物可耐受多种官能团，其 中供电子基团例如甲基、叔丁基的产物收率较好(表 2, Entries 1 和 3)，而位于邻位的甲氧基可能由于空间位阻 较大，只得到了 $43 \%$ 的 $3 \mathbf{b}$ (表 2, Entry 2). 对于强吸电子 基团例如硝基，反应基本没有产物生成(表 2, Entries 4). 对于苄基胺、甲基苄胺和苯乙胺，在最佳实验条件下， 均可以与 GVL 反应生成相对应的羟基酰胺 1e、1f 及 $\mathbf{~ g}$ (表 2, Entries 5 7). 其中甲基芐胺由于苄位甲基的存 在，对反应活性有一定的影响. 同样，2-呋喃甲胺也可 以与 GVL 反应生成相应的羟基酰胺化合物 $\mathbf{~ h}$ (表 2 , Entry 8), 这对于生物质平台分子的利用以及呋喃基药 物的开发具有较大意义. 基于前人报道的初级烷基胺的 优异活性，对环己胺底物进行了考察，以 $45 \%$ 的收率合 成了相应的酰胺类化合物 $1 \mathrm{i}$ (表 2, Entry 9). 考察了其他 内酯类化合物与苯胺的反应情况，进一步制备了具有长 支链羟基酰胺化合物 1j, 1k, 11(表 2, Entries 10１2). 令 人满意的是, 使用这三种六元内酯作为底物, 均可以得 到中等的产物收率( $66 \%$ \% $75 \%$ ). 
表 2 以 Zr-P-O 为催化剂制备羟基酰胺化合物 ${ }^{a}$

Table 2 Preparation of hydroxyamides with Zr-P-O as catalyst

\begin{tabular}{|c|c|c|c|c|}
\hline Entry & Amine & Lactone & Product & Yield/\% \\
\hline 1 & & & & 62 \\
\hline 2 & & & & 43 \\
\hline 3 & & & & 58 \\
\hline 4 & & & & n.r. \\
\hline 5 & & & & 64 \\
\hline 6 & N & & & 49 \\
\hline 7 & & & & 67 \\
\hline 8 & (1) & & & 43 \\
\hline 9 & & & & 45 \\
\hline 10 & & & & 66 \\
\hline 11 & & & & 72 \\
\hline 12 & & & & 75 \\
\hline
\end{tabular}

${ }^{a}$ Reaction conditions: lactone $(1 \mathrm{mmol})$, aniline $(1.1 \mathrm{mmol})$, catalyst $(\mathrm{Zr}-\mathrm{P}-\mathrm{O}, 100 \mathrm{mg})$, acetonitrile $(1 \mathrm{~mL}), 120{ }^{\circ} \mathrm{C}, 48 \mathrm{~h}$. 
进一步考察了不同取代基的芳香胺与 GVL 生成吡 咯烷酮类化合物的反应情况, 结果列在表 3 中.

首先，通过使用邻位、间位或对位不同取代基的苯 胺来考察反应的普适性. 给电子基团如甲基和甲氧基取 代的苯胺显示出与苯胺类似的反应性, 以中等偏上的产 率得到了相应的产物. 从结果可以看出, 甲基的取代位 置显著影响了胺与 GVL 的反应活性, 它们的反应活性 遵循以下顺序：对甲基苯胺 $>$ 间甲基苯胺 $>$ 邻甲基苯胺 (表 3, Entries 1 3). 同样, 甲氧基取代位置在邻位的苯 胺化合物的反应活性也较差, 仅以 $39 \%$ 的收率得到了产 物 2e(表 3, Entry 5), 这与邻位取代基造成的空间位阻有 关. 此外, 以苄基胺、苯乙胺为底物进行了考察, 在最佳 实验条件下均以适中的产物收率得到了吡咯烷酮类化 合物 2h 与 2i(表 3, Entries 8, 9).
根据实验结果及文献的报道 ${ }^{[14,11 \mathrm{c}, 31]}$, 提出了可能的 反应机理(Scheme 2). Zr-P-O 提供的酸性的环境促进了 氨基进攻内酯的羰基官能团，使得 GVL 发生开环反应， 进而形成羟基酰胺. 在更强的路易斯酸 $\mathrm{ZrOCl}_{2} \bullet 8 \mathrm{H}_{2} \mathrm{O}$ 的 催化下，羟基酰胺发生自身的脱水关环反应，最终得到 了吡咯烷酮化合物。

\section{2 结论}

综上所述, 研究了以 GVL 为模型底物的内酯类化 合物的还原胺化环合反应，在非均相催化剂 Zr-P-O 存 在下，最终的主产物为羟基酰胺类化合物，在强 Lewis 酸 $\mathrm{ZrOCl}_{2} \cdot 8 \mathrm{H}_{2} \mathrm{O}$ 的催化作用下, $\mathrm{GVL}$ 能够与胺类化合物 反应得到的羟基酰胺类中间体进一步环合得到吡咯烷

表 3 以 $\mathrm{ZrOCl}_{2} \bullet 8 \mathrm{H}_{2} \mathrm{O}$ 为催化剂制备吡咯烷酮化合物 ${ }^{a}$

Table 3 Preparation of pyrrolidones with $\mathrm{ZrOCl}_{2} \bullet 8 \mathrm{H}_{2} \mathrm{O}$ as catalyst

\begin{tabular}{|c|c|c|c|c|c|c|c|}
\hline Entry & Substrate & Product & Yield/\% & Entry & Substrate & Product & Yield/\% \\
\hline 1 & 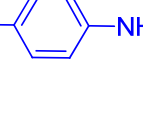 & $2 a$ & 65 & 6 & & $2 f$ & 53 \\
\hline 2 & & $2 b$ & 58 & 7 & 1 & $2 g$ & 41 \\
\hline 3 & & $2 c$ & 44 & 8 & & $2 \mathrm{~h}$ & 66 \\
\hline 4 & $-\mathrm{N}$ & $2 d$ & 62 & 9 & & $2 \mathbf{i}$ & 70 \\
\hline 5 & & $2 e$ & 39 & & & & \\
\hline
\end{tabular}

${ }^{a}$ Reaction conditions: GVL $(1 \mathrm{mmol})$, aniline $(1.1 \mathrm{mmol})$, catalyst $\left(\mathrm{ZrOCl}_{2} \bullet 8 \mathrm{H}_{2} \mathrm{O}, 50 \mathrm{mg}\right)$, acetonitrile $(1 \mathrm{~mL}), 120{ }^{\circ} \mathrm{C}, 48 \mathrm{~h}$.

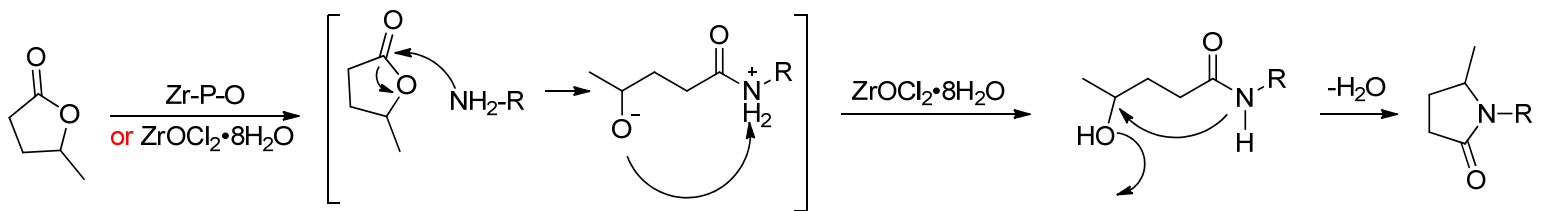

图式 2 可能的反应机理

Scheme 2 Possible reaction mechanism 
酮类化合物. 该催化体系条件温和, 操作简便, 是一种 具有广阔应用前景的合成吡咯烷酮类化合物的方法.

\section{3 实验部分}

\section{1 仪器与试剂}

催化剂 Zr-P-O 参照文献[26]制备, 其他试剂均为商 购. 核磁共振谱使用 Bruker Avance $400 \mathrm{MHz}$ 光谱仪(以 TMS 作为内标, $\mathrm{CDCl}_{3}$ 或 DMSO- $d_{6}$ 为溶剂)测定. 核磁共 振谱用 MestReNova 软件分析.

\section{$3.2 \mathrm{Zr}-\mathrm{P}-\mathrm{O}$ 催化剂的制备}

$\mathrm{A}$ 溶液: 将 $5.2 \mathrm{~g} \mathrm{ZrOCl}_{2} \cdot 8 \mathrm{H}_{2} \mathrm{O}(0.03 \mathrm{~mol})$ 加入到 16 $\mathrm{mL}$ 的去离子水中, 在搅拌下加热到 $70{ }^{\circ} \mathrm{C}$, 保温搅拌 10 min, 制得 $\mathrm{A}$ 溶液.

$\mathrm{B}$ 溶液: 将 $3.71 \mathrm{~g} \mathrm{NH}_{4} \mathrm{H}_{2} \mathrm{PO}_{4}(0.03 \mathrm{~mol})$ 加入到 32 $\mathrm{mL}$ 的去离子水中, 搅拌使其溶剂充分, 制得 $\mathrm{B}$ 溶液.

混合: 在剧烈摚拌下, 将 $\mathrm{B}$ 溶液逐渐滴加到 $\mathrm{A}$ 溶液 中, 加入完毕, 有大量沉淀生成, 继续在 $70{ }^{\circ} \mathrm{C}$ 保温搅拌 $1 \mathrm{~h}$. 将沉淀物过滤, 用 $100 \mathrm{~mL}$ 去离子水清洗 5 次, 并在 $100{ }^{\circ} \mathrm{C}$ 下干燥 $10 \mathrm{~h}$. 将干燥后的催化剂研磨后在 $400{ }^{\circ} \mathrm{C}$ 下煅烧 $3 \mathrm{~h}$.

\section{2 实验方法}

\subsection{1 制备羟基酰胺的代表性步骤}

将苯胺 $(1.1 \mathrm{mmol})$ 加入到耐压管中, 然后依次加入 乙腈 $(1 \mathrm{~mL}), \mathrm{GVL}$ (1 mmol), 以及 Zr-P-O 催化剂 $100 \mathrm{mg}$, 旋紧旋塞后, 在磁力摚拌 $(600 \mathrm{r} / \mathrm{min})$ 下将反应混合物加 热至 $120{ }^{\circ} \mathrm{C}$, 搅拌 $48 \mathrm{~h}$. 反应结束后, 冷却至室温, 过 滤除去 Zr-P-O 催化剂, 将反应液浓缩, 通过柱层析进行 分离得到 4-羟基- $N$-苯基戊酰胺(1): ${ }^{[32]} 120 \mathrm{mg}$, 白色固 体, 产率 62\%. m.p. 88 $89{ }^{\circ} \mathrm{C} ;{ }^{1} \mathrm{H}$ NMR $(400 \mathrm{MHz}$, $\left.\mathrm{CDCl}_{3}\right) \delta: 7.88(\mathrm{~s}, 1 \mathrm{H}), 7.50(\mathrm{~d}, J=7.8 \mathrm{~Hz}, 2 \mathrm{H}), 7.30(\mathrm{~s}$, 2H), $7.10(\mathrm{~d}, J=7.3 \mathrm{~Hz}, 1 \mathrm{H}), 3.95 \sim 3.84(\mathrm{~m}, 1 \mathrm{H}), 2.53$ (dd, $J=6.2,4.3 \mathrm{~Hz}, 2 \mathrm{H}), 1.97 \sim 1.88(\mathrm{~m}, 1 \mathrm{H}), 1.78(\mathrm{~s}, 1 \mathrm{H})$, 1.23 (s, 3H); ${ }^{13} \mathrm{C}$ NMR (101 MHz, $\left.\mathrm{CDCl}_{3}\right) \delta: 172.0,137.9$, 129.0, 124.3, 120.0, 67.6, 34.2, 23.8 .

产物 $\mathbf{1 a} \sim \mathbf{1 k}$ 的制备方法同产物 $\mathbf{1}$.

4-羟基- $N$-(对甲苯基)戊酰胺 (1a $)^{[36]:} 128 \mathrm{mg}$, 白色 固体, 产率 62\%. m.p. 202 203 ${ }^{\circ} \mathrm{C} ;{ }^{1} \mathrm{H}$ NMR $(400 \mathrm{MHz}$, $\left.\mathrm{CDCl}_{3}\right) \delta: 7.51(\mathrm{~s}, 1 \mathrm{H}), 7.38(\mathrm{~d}, J=8.3 \mathrm{~Hz}, 2 \mathrm{H}), 7.12(\mathrm{~d}$, $J=8.2 \mathrm{~Hz}, 2 \mathrm{H}$ ), 3.92 (ddd, $J=9.1,6.2,3.3 \mathrm{~Hz}, 1 \mathrm{H}), 2.52$ (td, $J=6.9,3.2 \mathrm{~Hz}, 2 \mathrm{H}), 2.31$ (s, 3H), 1.93 (dtd, $J=10.6$, 7.3, $3.5 \mathrm{~Hz}, 1 \mathrm{H}), 1.79$ (dt, $J=14.4,7.4 \mathrm{~Hz}, 1 \mathrm{H}), 1.24$ (d, $J=6.3 \mathrm{~Hz}, 3 \mathrm{H}) ;{ }^{13} \mathrm{C}$ NMR $\left(101 \mathrm{MHz}, \mathrm{CDCl}_{3}\right) \delta: 171.7$, 135.2, 134.0, 129.5, 120.0, 67.6, 34.2, 23.9, 20.9.

4-羟基- $N$-(2-甲氧基苯基)戊酰胺(1b): $96 \mathrm{mg}$, 白色
固体, 产率 43\%. m.p. $224 \sim 225{ }^{\circ} \mathrm{C} ;{ }^{1} \mathrm{H}$ NMR $(400 \mathrm{MHz}$, $\left.\mathrm{CDCl}_{3}\right) \delta: 8.35(\mathrm{~d}, J=7.8 \mathrm{~Hz}, 1 \mathrm{H}), 7.88(\mathrm{~s}, 1 \mathrm{H}), 7.04(\mathrm{t}$, $J=7.5 \mathrm{~Hz}, 1 \mathrm{H}), 6.96(\mathrm{t}, J=7.7 \mathrm{~Hz}, 1 \mathrm{H}), 6.88(\mathrm{~d}, J=8.4$ $\mathrm{Hz}, 1 \mathrm{H}), 3.97 \sim 3.90(\mathrm{~m}, 1 \mathrm{H}), 3.87(\mathrm{~d}, J=11.5 \mathrm{~Hz}, 3 \mathrm{H})$, $2.57(\mathrm{t}, J=7.0 \mathrm{~Hz}, 2 \mathrm{H}), 1.93(\mathrm{ddd}, J=14.1,7.1,3.6 \mathrm{~Hz}$, $1 \mathrm{H}), 1.84 \sim 1.78(\mathrm{~m}, 1 \mathrm{H}), 1.25(\mathrm{~d}, J=5.9 \mathrm{~Hz}, 3 \mathrm{H}) ;{ }^{13} \mathrm{C}$ NMR $\left(101 \mathrm{MHz}, \mathrm{CDCl}_{3}\right) \delta: 139.0,120.1,118.6 \sim 118.4$, 108.6, 66.5, 54.6, 33.2, 28.7, 22.8 .

$\mathrm{N}$-(4-(叔丁基)苯基)-4-羟基戊酰胺(1c)：144 mg，白 色固体, 产率 58\%. m.p. $238 \sim 239{ }^{\circ} \mathrm{C} ;{ }^{1} \mathrm{H}$ NMR $(400$ $\left.\mathrm{MHz}, \mathrm{CDCl}_{3}\right) \delta: 7.35$ (d, $\left.J=8.6 \mathrm{~Hz}, 2 \mathrm{H}\right), 7.27$ (d, $J=8.5$ $\mathrm{Hz}, 2 \mathrm{H}), 3.85$ (s, 1H), $2.46(\mathrm{dd}, J=8.7,5.4 \mathrm{~Hz}, 2 \mathrm{H})$, $1.92 \sim 1.83(\mathrm{~m}, 1 \mathrm{H}), 1.71(\mathrm{dd}, J=14.6,8.0 \mathrm{~Hz}, 1 \mathrm{H}), 1.23$ (s, 9H), 1.19 (s, 3H); ${ }^{13} \mathrm{C}$ NMR (101 MHz, $\left.\mathrm{CDCl}_{3}\right) \delta$ : 170.7, 146.3, 134.1, 124.8, 118.7, 66.6, 33.1, 30.3, 28.7, 22.8 .

$N$-芐基-4-羟基戊酰胺(1e $)^{[37]}: 132 \mathrm{mg}$, 白色固体, 产率 64\%. m.p. $189 \sim 190{ }^{\circ} \mathrm{C} ;{ }^{1} \mathrm{H}$ NMR $(400 \mathrm{MHz}$, $\left.\mathrm{CDCl}_{3}\right) \delta: 7.37 \sim 7.29(\mathrm{~m}, 2 \mathrm{H}), 7.27(\mathrm{dd}, J=7.8,5.5 \mathrm{~Hz}$, $3 \mathrm{H}), 4.42(\mathrm{t}, J=5.2 \mathrm{~Hz}, 2 \mathrm{H}), 3.89 \sim 3.79(\mathrm{~m}, 1 \mathrm{H}), 2.38(\mathrm{td}$, $J=6.7,3.2 \mathrm{~Hz}, 2 \mathrm{H}$ ), 1.84 (dddd, $J=14.1,10.7,7.3,3.8$ $\mathrm{Hz}, 1 \mathrm{H}), 1.71$ (tdd, $J=14.7,8.9,5.6 \mathrm{~Hz}, 1 \mathrm{H}), 1.20$ (dd, $J=$ 6.2, $3.7 \mathrm{~Hz}, 3 \mathrm{H}) ;{ }^{13} \mathrm{C}$ NMR $\left(101 \mathrm{MHz}, \mathrm{CDCl}_{3}\right) \delta: 173.6$, 138.1, 128.7, 127.8, 127.6, 67.5, 43.7, 34.3, 33.2, 23.7.

4-羟基- $N$-(1-苯乙基)戊酰胺(1f): $108 \mathrm{mg}$, 白色固体, 产率 $49 \%$. m.p. $186 \sim 187{ }^{\circ} \mathrm{C} ;{ }^{1} \mathrm{H}$ NMR $(400 \mathrm{MHz}$, $\left.\mathrm{CDCl}_{3}\right) \delta: 7.35 \sim 7.26(\mathrm{~m}, 4 \mathrm{H}), 5.08(\mathrm{dt}, J=11.2,5.5 \mathrm{~Hz}$, $1 \mathrm{H}), 3.77(\mathrm{~s}, 1 \mathrm{H}), 2.36 \sim 2.24(\mathrm{~m}, 2 \mathrm{H}), 1.84 \sim 1.72(\mathrm{~m}$, $1 \mathrm{H}$ ), 1.66 (ddd, $J=14.4,9.4,2.5 \mathrm{~Hz}, 1 \mathrm{H}), 1.50 \sim 1.39$ (m, $3 \mathrm{H}), 1.16(\mathrm{dd}, J=6.1,3.4 \mathrm{~Hz}, 3 \mathrm{H}) ;{ }^{13} \mathrm{C}$ NMR $(101 \mathrm{MHz}$, $\left.\mathrm{CDCl}_{3}\right) \delta: 172.9,143.2,128.6,127.3,126.1,67.4,48.9$, 34.4, 33.2, 23.6, 21.8 .

4-羟基- $N$-苯乙基戊酰胺 $(\mathbf{1 g})^{[38]}: 148 \mathrm{mg}$, 白色固体, 产率 67\%. m.p. 201 202 ${ }^{\circ} \mathrm{C}$; ${ }^{1} \mathrm{H}$ NMR $(400 \mathrm{MHz}$, $\left.\mathrm{CDCl}_{3}\right) \delta: 7.31(\mathrm{t}, J=7.3 \mathrm{~Hz}, 2 \mathrm{H}), 7.24(\mathrm{~d}, J=7.2 \mathrm{~Hz}, 1 \mathrm{H})$, $7.20(\mathrm{t}, J=6.2 \mathrm{~Hz}, 2 \mathrm{H}), 5.83(\mathrm{~s}, 1 \mathrm{H}), 3.83 \sim 3.74(\mathrm{~m}, 1 \mathrm{H})$, $3.52(\mathrm{dd}, J=13.0,6.8 \mathrm{~Hz}, 2 \mathrm{H}), 2.82(\mathrm{t}, J=7.0 \mathrm{~Hz}, 2 \mathrm{H})$, 2.29 (dd, $J=10.4,3.9 \mathrm{~Hz}, 2 \mathrm{H}), 1.79(\mathrm{dtd}, J=14.1,7.2,3.5$ $\mathrm{Hz}, 1 \mathrm{H}), 1.65(\mathrm{dt}, J=14.7,6.8 \mathrm{~Hz}, 1 \mathrm{H}), 1.18(\mathrm{~d}, J=6.2$ $\mathrm{Hz}, 3 \mathrm{H}) ;{ }^{13} \mathrm{C}$ NMR $\left(101 \mathrm{MHz}, \mathrm{CDCl}_{3}\right) \delta: 172.7,137.8$, $127.7,125.5,66.4,39.6,34.5,33.2,32.3,22.6$.

$\mathrm{N}$-(呋喃-2-基甲基)-4-羊基戊酰胺(1h): $85 \mathrm{mg}$, 淡黄 色固体, 产率 43\%. m.p. 196 197 ${ }^{\circ} \mathrm{C} ;{ }^{1} \mathrm{H}$ NMR (400 $\left.\mathrm{MHz}, \mathrm{CDCl}_{3}\right) \delta: 7.35(\mathrm{~s}, 1 \mathrm{H}), 6.31(\mathrm{~s}, 1 \mathrm{H}), 6.22(\mathrm{~d}, J=2.9$ $\mathrm{Hz}, 1 \mathrm{H}), 4.41(\mathrm{~d}, J=5.5 \mathrm{~Hz}, 2 \mathrm{H}), 3.87 \sim 3.78(\mathrm{~m}, 1 \mathrm{H})$, 
$3.07(\mathrm{~s}, 1 \mathrm{H}), 2.39 \sim 2.33(\mathrm{~m}, 2 \mathrm{H}), 1.83(\mathrm{dtd}, J=10.6,7.1$, $3.6 \mathrm{~Hz}, 1 \mathrm{H}), 1.69(\mathrm{td}, J=14.8,6.9 \mathrm{~Hz}, 1 \mathrm{H}), 1.19$ (d, $J=6.2$ $\mathrm{Hz}, 3 \mathrm{H}) ;{ }^{13} \mathrm{C}$ NMR (101 MHz, $\left.\mathrm{CDCl}_{3}\right) \delta: 172.5,150.2$, 141.2, 109.5, 106.5, 66.4, 35.6, 33.2, 32.0, 22.6.

$\mathrm{N}$-环已基-4-差基戊酰胺(1i): $90 \mathrm{mg}$, 白色固体, 产 率 45\%. m.p. $159 \sim 160{ }^{\circ} \mathrm{C}$; ${ }^{1} \mathrm{H}$ NMR $\left(400 \mathrm{MHz}, \mathrm{CDCl}_{3}\right)$ $\delta: 5.82(\mathrm{~s}, 1 \mathrm{H}), 3.89 \sim 3.79(\mathrm{~m}, 1 \mathrm{H}), 3.80 \sim 3.68(\mathrm{~m}, 1 \mathrm{H})$, $2.39 \sim 2.26(\mathrm{~m}, 2 \mathrm{H}), 1.90(\mathrm{~d}, J=10.2 \mathrm{~Hz}, 2 \mathrm{H}), 1.81$ (dtd, $J=10.4,7.0,3.3 \mathrm{~Hz}, 1 \mathrm{H}), 1.77 \sim 1.65(\mathrm{~m}, 3 \mathrm{H}), 1.62(\mathrm{dd}$, $J=9.9,4.5 \mathrm{~Hz}, 1 \mathrm{H}), 1.35$ (ddd, $J=33.7,17.7,13.6 \mathrm{~Hz}$, $3 \mathrm{H}), 1.20(\mathrm{~d}, J=6.2 \mathrm{~Hz}, 3 \mathrm{H}), 1.13(\mathrm{dd}, J=16.8,7.3 \mathrm{~Hz}$, $2 \mathrm{H}) ;{ }^{13} \mathrm{C}$ NMR $\left(101 \mathrm{MHz}, \mathrm{CDCl}_{3}\right) \delta: 171.8,66.4,47.3$, 33.4, 32.5, 32.1, 24.5, 23.8, 22.6 .

2-(2-(羟甲基)苯基)- $\mathrm{N}$-苯基乙酰胺(1j) $)^{[12 b]: ~} 159 \mathrm{mg}$, 白色固体, 产率 66\%. m.p. $277 \sim 278{ }^{\circ} \mathrm{C} ;{ }^{1} \mathrm{H}$ NMR (400 $\left.\mathrm{MHz}, \mathrm{CDCl}_{3}\right) \delta: 8.35(\mathrm{~s}, 1 \mathrm{H}), 7.43(\mathrm{t}, J=9.2 \mathrm{~Hz}, 3 \mathrm{H})$, $7.39 \sim 7.29(\mathrm{~m}, 3 \mathrm{H}), 7.29 \sim 7.27(\mathrm{~m}, 1 \mathrm{H}), 7.23(\mathrm{~s}, 1 \mathrm{H})$, 7.05 (t, $J=7.4 \mathrm{~Hz}, 1 \mathrm{H}), 4.78$ (s, 2H), 3.79 (s, 2H), 3.11 (s, $1 \mathrm{H}) ;{ }^{13} \mathrm{C}$ NMR (101 MHz, $\left.\mathrm{CDCl}_{3}\right) \delta: 138.4,138.0,134.3$, 130.7, 130.4, 129.0, 127.9, 124.2, 119.6, 64.3, 42.2.

4-羟基- $N$-苯基壬酰胺 $(\mathbf{1 k})^{[33]}$ : $179 \mathrm{mg}$, 白色固体, 产率 72\%. m.p. 99 100 ${ }^{\circ} \mathrm{C} ;{ }^{1} \mathrm{H}$ NMR $\left(400 \mathrm{MHz}, \mathrm{CDCl}_{3}\right)$ $\delta: 7.52$ (d, $J=7.9 \mathrm{~Hz}, 2 \mathrm{H}), 7.30$ (t, $J=7.6 \mathrm{~Hz}, 2 \mathrm{H}), 7.09$ (t, $J=7.3 \mathrm{~Hz}, 1 \mathrm{H}), 3.63(\mathrm{~s}, 1 \mathrm{H}), 2.41$ (t, $J=7.2 \mathrm{~Hz}, 2 \mathrm{H}), 1.84$ (s, 2H), $1.59 \sim 1.46(\mathrm{~m}, 2 \mathrm{H}), 1.44$ (d, $J=5.3 \mathrm{~Hz}, 2 \mathrm{H}), 1.29$ (d, $J=5.0 \mathrm{~Hz}, 4 \mathrm{H}), 0.88(\mathrm{t}, J=6.7 \mathrm{~Hz}, 3 \mathrm{H}) ;{ }^{13} \mathrm{C}$ NMR $(101$ $\left.\mathrm{MHz}, \mathrm{CDCl}_{3}\right) \delta: 171.7,138.0,129.0,124.2,119.9,71.6$, $37.6,37.3,36.3,31.9,25.4,22.6,21.7,14.1$.

4-羟基- $N$-苯基十一碳酰胺 $(\mathbf{1 1})^{[33]}: 208 \mathrm{mg}$, 白色固 体, 产率 75\%. m.p. 106 107 ${ }^{\circ} \mathrm{C}$; ${ }^{1} \mathrm{H}$ NMR $(400 \mathrm{MHz}$, $\left.\mathrm{CDCl}_{3}\right) \delta: 7.80(\mathrm{~s}, 1 \mathrm{H}), 7.52(\mathrm{~d}, J=6.9 \mathrm{~Hz}, 2 \mathrm{H}), 7.29$ (s, 2H), 7.09 (s, 1H), 3.62 (s, 1H), 2.40 (s, 2H), 2.13 (s, 1H), $1.84(\mathrm{~s}, 2 \mathrm{H}), 1.63 \sim 1.47(\mathrm{~m}, 2 \mathrm{H}), 1.43(\mathrm{~s}, 2 \mathrm{H}), 1.27(\mathrm{~s}$, $8 \mathrm{H}), 0.88(\mathrm{~s}, 3 \mathrm{H}) ;{ }^{13} \mathrm{C}$ NMR $\left(101 \mathrm{MHz}, \mathrm{CDCl}_{3}\right) \delta: 170.7$, $137.0,127.9,123.2,118.9,70.6,36.6,36.3,35.3,30.8$, 28.6, 28.3, 24.7, 21.6, 20.7, 13.1 .

\subsection{2 制备吡咯烷酮的代表性步骤}

将苯胺 $(1.1 \mathrm{mmol})$ 加入到耐压管中, 然后依次加入 乙腈 $1 \mathrm{~mL}, \mathrm{GVL}(1 \mathrm{mmol})$, 以及 $\mathrm{ZrOCl}_{2} \bullet 8 \mathrm{H}_{2} \mathrm{O}$ 催化剂 50 $\mathrm{mg}$, 旋紧旋塞后, 在磁力摚拌 $(600 \mathrm{r} / \mathrm{min})$ 下, 将反应混 合物加热至 $120{ }^{\circ} \mathrm{C}$, 搅拌 $48 \mathrm{~h}$. 反应结束后, 冷却至室 温, 加入 $10 \mathrm{~mL}$ 水, 使用乙酸乙酯萃取三次, 萃取液使 用饱和氯化钠溶液洗, 然后将萃取液浓缩, 通过柱层析 进行分离得到 5-甲基-1-苯基-2-吡咯烷酮(2) ${ }^{[34]} 124 \mathrm{mg}$, 白色固体, 产率 $71 \%$. m.p. $81 \sim 82{ }^{\circ} \mathrm{C} ;{ }^{1} \mathrm{H}$ NMR (400
$\left.\mathrm{MHz}, \mathrm{CDCl}_{3}\right) \delta: 7.31(\mathrm{~d}, J=6.5 \mathrm{~Hz}, 4 \mathrm{H}), 7.14(\mathrm{~d}, J=6.3$ $\mathrm{Hz}, 1 \mathrm{H}), 4.23(\mathrm{~d}, J=6.4 \mathrm{~Hz}, 1 \mathrm{H}), 2.62 \sim 2.45(\mathrm{~m}, 2 \mathrm{H})$, $2.35 \sim 2.25(\mathrm{~m}, 1 \mathrm{H}), 1.74 \sim 1.63(\mathrm{~m}, 1 \mathrm{H}), 1.14(\mathrm{~d}, J=6.2$ $\mathrm{Hz}, 3 \mathrm{H}) ;{ }^{13} \mathrm{C}$ NMR $\left(101 \mathrm{MHz}, \mathrm{CDCl}_{3}\right) \delta: 173.2,136.5$, 128.0, 124.7, 123.0, 54.6, 30.4, 25.7, 19.2.

产物 $\mathbf{2} \mathbf{a} \sim \mathbf{2} \mathbf{j}$ 的制备方法同产物 $\mathbf{2}$.

5-甲基-1-(对甲苯基)吡咯烷-2-酮(2a) ${ }^{[34]}: 123 \mathrm{mg}$, 白色固体, 产率 65\%. m.p. 105 106 ${ }^{\circ} \mathrm{C} ;{ }^{1} \mathrm{H}$ NMR $(400$ $\left.\mathrm{MHz}, \mathrm{CDCl}_{3}\right) \delta: 7.21(\mathrm{q}, J=8.5 \mathrm{~Hz}, 4 \mathrm{H}), 4.30 \sim 4.18(\mathrm{~m}$, $1 \mathrm{H}), 2.68 \sim 2.46(\mathrm{~m}, 2 \mathrm{H}), 2.40 \sim 2.30(\mathrm{~m}, 4 \mathrm{H}), 1.74$ (dddd, $J=13.0,9.4,7.3,5.9 \mathrm{~Hz}, 1 \mathrm{H}), 1.18(\mathrm{~d}, J=6.2 \mathrm{~Hz}, 3 \mathrm{H}) ;{ }^{13} \mathrm{C}$ NMR $\left(101 \mathrm{MHz}, \mathrm{CDCl}_{3}\right) \delta: 174.3,135.7,134.9,129.6$, $124.3,55.8,31.3,26.8,21.0,20.2$.

5-甲基-1-(间甲基)吡咯烷-2-酮(2b) ${ }^{[30]}: 109 \mathrm{mg}$ ，白 色固体 [CAS: 1843259-88-0], 产率 58\%. m.p. 105 $106{ }^{\circ} \mathrm{C} ;{ }^{1} \mathrm{H}$ NMR $\left(400 \mathrm{MHz}, \mathrm{CDCl}_{3}\right) \delta: 7.27$ (t, $J=7.8 \mathrm{~Hz}$, 1H), 7.19 (s, 1H), $7.12(\mathrm{~d}, J=8.0 \mathrm{~Hz}, 1 \mathrm{H}), 7.02$ (d, $J=7.5$ $\mathrm{Hz}, 1 \mathrm{H}), 4.34 \sim 4.19(\mathrm{~m}, 1 \mathrm{H}), 2.63 \sim 2.51(\mathrm{~m}, 2 \mathrm{H}), 2.39 \sim$ $2.33(\mathrm{~m}, 4 \mathrm{H}), 1.78 \sim 1.68(\mathrm{~m}, 1 \mathrm{H}), 1.19(\mathrm{~d}, J=6.2 \mathrm{~Hz}$, $3 \mathrm{H}) ;{ }^{13} \mathrm{C}$ NMR $\left(101 \mathrm{MHz}, \mathrm{CDCl}_{3}\right) \delta: 174.3,138.9,137.5$, $128.8,126.8,125.1,121.3,55.8,31.4,26.8,21.5,20.2$.

5-甲基-1-(邻甲苯基)吡咯烷-2-酮(2c) ${ }^{[30]}: 83 \mathrm{mg}$, 白 色固体, 产率 44\%. m.p. $105 \sim 106{ }^{\circ} \mathrm{C} ;{ }^{1} \mathrm{H}$ NMR (400 $\left.\mathrm{MHz}, \mathrm{CDCl}_{3}\right) \delta: 7.29 \sim 7.26(\mathrm{~m}, 1 \mathrm{H}), 7.25 \sim 7.18(\mathrm{~m}, 2 \mathrm{H})$, $7.09 \sim 7.03(\mathrm{~m}, 1 \mathrm{H}), 4.08(\mathrm{~s}, 1 \mathrm{H}), 2.62 \sim 2.54(\mathrm{~m}, 2 \mathrm{H})$, $2.44 \sim 2.37(\mathrm{~m}, 1 \mathrm{H}), 2.23(\mathrm{~s}, 3 \mathrm{H}), 1.84 \sim 1.76(\mathrm{~m}, 1 \mathrm{H})$, $1.12(\mathrm{~d}, J=6.3 \mathrm{~Hz}, 3 \mathrm{H}) ;{ }^{13} \mathrm{C}$ NMR $\left(101 \mathrm{MHz}, \mathrm{CDCl}_{3}\right) \delta$ : 174.3, 136.4, 136.1, 131.2, 127.9, 126.7, 56.9, 30.9, 27.9, $20.3,18.1$.

1-(4-甲氧基苯基)-5-甲基吡咯烷-2-酮(2d ${ }^{[34]}$ : 127 $\mathrm{mg}$, 白色固体, 产率 $62 \%$. m.p. $127 \sim 128{ }^{\circ} \mathrm{C} ;{ }^{1} \mathrm{H}$ NMR $\left(400 \mathrm{MHz}, \mathrm{CDCl}_{3}\right) \delta: 7.23(\mathrm{~d}, J=8.9 \mathrm{~Hz}, 2 \mathrm{H}), 6.95 \sim 6.88$ $(\mathrm{m}, 2 \mathrm{H}), 4.23 \sim 4.13(\mathrm{~m}, 1 \mathrm{H}), 3.80(\mathrm{t}, J=2.0 \mathrm{~Hz}, 3 \mathrm{H})$, $2.66 \sim 2.47(\mathrm{~m}, 2 \mathrm{H}), 2.41 \sim 2.31(\mathrm{~m}, 1 \mathrm{H}), 1.79 \sim 1.67(\mathrm{~m}$, $1 \mathrm{H}), 1.20 \sim 1.14(\mathrm{~m}, 3 \mathrm{H}) ;{ }^{13} \mathrm{C} \mathrm{NMR}\left(101 \mathrm{MHz}, \mathrm{CDCl}_{3}\right) \delta$ : $174.4,157.7,130.4,126.1,114.3,56.2,55.5,31.2,26.8$, 20.3 .

1-(2-甲氧基苯基)-5-甲基吡咯烷-2-酮(2e) ${ }^{[16 \mathrm{hb}}: 80$ $\mathrm{mg}$, 白色固体, 产率 $39 \%$. m.p. $127 \sim 128{ }^{\circ} \mathrm{C} ;{ }^{1} \mathrm{H}$ NMR $\left(400 \mathrm{MHz}, \mathrm{CDCl}_{3}\right) \delta: 7.31 \sim 7.26(\mathrm{~m}, 1 \mathrm{H}), 7.15(\mathrm{~d}, J=7.7$ $\mathrm{Hz}, 1 \mathrm{H}), 6.97$ (dd, $J=12.6,7.9 \mathrm{~Hz}, 2 \mathrm{H}), 4.25 \sim 4.10(\mathrm{~m}$, $1 \mathrm{H}), 3.82(\mathrm{~s}, 3 \mathrm{H}), 2.58 \sim 2.53(\mathrm{~m}, 2 \mathrm{H}), 2.40 \sim 2.32(\mathrm{~m}$, 1H), 1.73 (ddd, $J=9.3,7.1,3.6 \mathrm{~Hz}, 1 \mathrm{H}), 1.07$ (d, $J=6.3$ $\mathrm{Hz}, 3 \mathrm{H}) ;{ }^{13} \mathrm{C}$ NMR (101 MHz, $\left.\mathrm{CDCl}_{3}\right) \delta: 175.2,155.3$, $130.2,128.9,125.8,120.8,112.0,55.9,55.6,30.9,27.7$, 
20.4 .

1-(3-氯苯基)-5-甲基吡咯烷-2-酮(2f ${ }^{[16 \mathrm{~h}]}$ : $110 \mathrm{mg}$, 白色固体, 产率 53\%. m.p. 124 $125{ }^{\circ} \mathrm{C} ;{ }^{1} \mathrm{H}$ NMR (400 $\left.\mathrm{MHz}, \mathrm{CDCl}_{3}\right) \delta: 7.45(\mathrm{~s}, 1 \mathrm{H}), 7.31(\mathrm{~d}, J=5.3 \mathrm{~Hz}, 2 \mathrm{H})$, $7.21 \sim 7.14(\mathrm{~m}, 1 \mathrm{H}), 4.35 \sim 4.25(\mathrm{~m}, 1 \mathrm{H}), 2.65 \sim 2.53(\mathrm{~m}$, $2 \mathrm{H}), 2.40 \sim 2.34(\mathrm{~m}, 1 \mathrm{H}), 1.80 \sim 1.73(\mathrm{~m}, 1 \mathrm{H}), 1.22(\mathrm{~d}, J=$ $6.2 \mathrm{~Hz}, 3 \mathrm{H}) ;{ }^{13} \mathrm{C}$ NMR $\left(101 \mathrm{MHz}, \mathrm{CDCl}_{3}\right) \delta: 173.3,137.8$, 133.5, 128.9, 124.6, 122.6, 120.6, 54.4, 30.3, 25.5, 19.0.

1-(4-差基苯基)-5-甲基吡咯烷-2-酮(2g) ${ }^{[16 \mathrm{~h}]}: 78 \mathrm{mg}$, 白色固体，产率 41\%. m.p. 193 $194{ }^{\circ} \mathrm{C} ;{ }^{1} \mathrm{H}$ NMR (400 $\left.\mathrm{MHz}, \mathrm{CDCl}_{3}\right) \delta: 6.94(\mathrm{~d}, J=8.6 \mathrm{~Hz}, 2 \mathrm{H}), 6.64(\mathrm{~d}, J=8.7$ $\mathrm{Hz}, 2 \mathrm{H}), 4.15 \sim 4.03(\mathrm{~m}, 1 \mathrm{H}), 2.69 \sim 2.50(\mathrm{~m}, 2 \mathrm{H}), 2.44 \sim$ $2.31(\mathrm{~m}, 1 \mathrm{H}), 1.80 \sim 1.68(\mathrm{~m}, 1 \mathrm{H}), 1.14(\mathrm{~d}, J=6.3 \mathrm{~Hz}$, $3 \mathrm{H}) ;{ }^{13} \mathrm{C}$ NMR (101 MHz, $\left.\mathrm{CDCl}_{3}\right) \delta: 175.6,155.6,128.6$, 127.1, 116.6, 57.3, 31.2, 26.9, 20.4.

1-苄基-5-甲基吡咯烷-2-酮(2h) ${ }^{[35]}: 125 \mathrm{mg}$, 白色固 体, 产率 66\%. m.p. 152 153 ${ }^{\circ} \mathrm{C} ;{ }^{1} \mathrm{H}$ NMR $(400 \mathrm{MHz}$, $\left.\mathrm{CDCl}_{3}\right) \delta: 7.29(\mathrm{ddd}, J=13.2,9.1,4.6 \mathrm{~Hz}, 5 \mathrm{H}), 4.40(\mathrm{t}, J=$ $5.9 \mathrm{~Hz}, 2 \mathrm{H}), 3.82$ (ddd, $J=9.3,7.8,4.8 \mathrm{~Hz}, 1 \mathrm{H}), 2.36$ (td, $J=6.9,1.9 \mathrm{~Hz}, 2 \mathrm{H}), 1.82(\mathrm{dtd}, J=14.2,7.1,3.5 \mathrm{~Hz}, 1 \mathrm{H})$, 1.69 (dt, $J=14.6,6.8 \mathrm{~Hz}, 1 \mathrm{H}), 1.19$ (t, $J=5.3 \mathrm{~Hz}, 3 \mathrm{H}) ;{ }^{13} \mathrm{C}$ NMR (101 MHz, $\left.\mathrm{CDCl}_{3}\right) \delta: 173.7,138.2,128.7,127.8$, 127.5, 67.4, 43.7, 34.3, 33.2, 29.7, 23.6.

5-甲基-1-苯乙基吡咯烷-2-酮(2i) ${ }^{[16 e]}: 142 \mathrm{mg}$, 白色 固体, 产率 70\%. m.p. $104 \sim 105{ }^{\circ} \mathrm{C} ;{ }^{1} \mathrm{H}$ NMR $(400 \mathrm{MHz}$, $\left.\mathrm{CDCl}_{3}\right) \delta: 7.32(\mathrm{t}, J=7.3 \mathrm{~Hz}, 2 \mathrm{H}), 7.25(\mathrm{~d}, J=7.5 \mathrm{~Hz}, 1 \mathrm{H})$, $7.21(\mathrm{t}, J=6.5 \mathrm{~Hz}, 2 \mathrm{H}), 3.86 \sim 3.75(\mathrm{~m}, 1 \mathrm{H}), 3.53(\mathrm{dd}, J=$ 11.6, $5.7 \mathrm{~Hz}, 2 \mathrm{H}), 2.83(\mathrm{t}, J=6.9 \mathrm{~Hz}, 2 \mathrm{H}), 2.38 \sim 2.27(\mathrm{~m}$, $2 \mathrm{H}), 1.85 \sim 1.74(\mathrm{~m}, 1 \mathrm{H}), 1.66(\mathrm{td}, J=14.5,7.0 \mathrm{~Hz}, 1 \mathrm{H})$, $1.19(\mathrm{~d}, J=6.2 \mathrm{~Hz}, 3 \mathrm{H}) ;{ }^{13} \mathrm{C} \mathrm{NMR}\left(101 \mathrm{MHz}, \mathrm{CDCl}_{3}\right) \delta$ : $172.6,137.8,127.7,125.6,66.5,39.6,34.5,33.2,32.3$, 22.6 .

1,1'-([1,1'- 联苯 ]-4,4'-二基) 双 (5-甲基吡咯烷 -2酮)(2j): $195 \mathrm{mg}$, 白色固体，产率 56\%. m.p. 372 $373{ }^{\circ} \mathrm{C} ;{ }^{1} \mathrm{H}$ NMR (400 MHz, $\left.\mathrm{CDCl}_{3}\right) \delta: 7.60$ (d, $J=8.4$ $\mathrm{Hz}, 4 \mathrm{H}), 7.45$ (d, $J=8.5 \mathrm{~Hz}, 4 \mathrm{H}), 4.34$ (dt, $J=12.5,6.3$ $\mathrm{Hz}, 2 \mathrm{H}), 2.72 \sim 2.51(\mathrm{~m}, 4 \mathrm{H}), 2.45 \sim 2.34(\mathrm{~m}, 2 \mathrm{H}), 1.82 \sim$ $1.74(\mathrm{~m}, 2 \mathrm{H}), 1.26(\mathrm{~d}, J=6.2 \mathrm{~Hz}, 6 \mathrm{H}) ;{ }^{13} \mathrm{C} \mathrm{NMR}(101$ $\left.\mathrm{MHz}, \mathrm{CDCl}_{3}\right) \delta: 174.3,137.8,136.8,127.5,124.1,55.6$, 31.4, 26.7, 20.2.

辅助材料(Supporting Information) 本文涉及到的化 合物的 ${ }^{1} \mathrm{H}$ NMR 和 ${ }^{13} \mathrm{C}$ NMR 谱图. 这些材料可以免费从 本刊网站(http://sioc-journal.cn/)上下载.

\section{References}

[1] (a) Corma, A.; Iborra, S.; Velty, A. Chem. Rev. 2007, 107, 2411. (b) Gong, B.-X.; Yan, L.; Chen, M.-Y.; Deng, J.; Fu, Y. Chin. J. Org. Chem. 2017, 37, 3170 (in Chinese).

(輁宝祥, 严龙, 陈蒙远, 邓晋, 傅尧, 有机化学, 2017, 37, 3170.) (c) Yang, Z.; Fu, Y.; Guo, Q.-X. Chin. J. Org. Chem. 2014, 34, 273 (in Chinese).

(杨珍, 傅尧, 郭庆祥, 有机化学, 2014, 34, 273.)

(d) Wang, H.-J.; Zhao, Y.; Wang, C.; Fu, Y.; Guo, Q.-X. Acta Chim. Sinica 2009, 67, 893 (in Chinese).

(王华静, 赵岩, 王晨, 傅尧, 郭庆祥, 化学学报, 2009, 67, 893.) (e) Li, J.; Huang, Y.-B.; Guo, Q.-X.; Fu, Y. Acta Chim. Sinica 2014, 72,1223 (in Chinese)

(李江, 黄耀兵, 郭庆祥, 傅尧, 化学学报, 2014, 72, 1223.)

(f) Xu, J.; Fan, W.-G.; Popowycz, F.; Queneau, Y.; Gu, Y.-L. Chin. J. Org. Chem. 2019, 39, 2131 (in Chinese).

(徐静, 范维刚, 波波维奇・弗洛伦斯, 葛诺伊夫, 顾彦龙, 有机 化学, 2019, 39, 2131.)

[2] (a) Paul, S. F. US 5697987, 1997.

(b) Serrano-Ruiz, J. C.; Wang, D.; Dumesic, J. A. Green Chem. 2010, $12,574$.

[3] Geilen, F. M.; Engendahl, B.; Harwardt, A.; Marquardt, W.; Klankermayer, J.; Leitner, W. Angew. Chem., Int. Ed. 2010, 49, 5510 .

[4] Mehdi, H.; Fábos, V.; Tuba, R.; Bodor, A.; Mika, L. T.; Horváth, I. T. Top. Catal. 2008, $48,49$.

[5] Lange, J. P.; Price, R.; Ayoub, P. M.; Louis, J.; Petrus, L.; Clarke, L.; Gosselink, H. Angew. Chem., Int. Ed. 2010, 49, 4479.

[6] (a) Bond, J. Q.; Alonso, D. M.; Wang, D.; West, R. M.; Dumesic, J. A. Science 2010, 327, 1110.

(b) Bond, J. Q.; Martin Alonso, D.; West, R. M.; Dumesic, J. A. Langmuir 2010, 26, 16291.

[7] Lange, J. P.; Vestering, J. Z.; Haan, R. J. Chem. Commun. 2007, 33, 3488 .

[8] (a) Fieser, M.; Fieser, L. F.; Toromanoff, E.; Hirata, Y.; Heymann, H.; Tefft, M.; Bhattacharya, S. J. Am. Chem. Soc. 1956, 78, 2825. (b) Newkome, G. R.; Baker, G. R.; Saunders, M.J.; Russo, P. S.; Gupta, V. K.; Yao, Z.; Miller, J. E.; Bouillion, K. J. Am. Chem. Soc. 1986, 108, 752 .

(c) Newkome, G. R.; Yao, Z.; Baker, G. R.; Gupta, V. K.; Russo, P. S.; Saunders, M. J. J. Am. Chem. Soc. 1986, 108, 849.

[9] (a) Rodgers, S. J.; Ng, C. Y.; Raymond, K. N. J. Am. Chem. Soc. 1985, 107, 4094.

(b) Collins, T. J.; Coots, R. J.; Furutani, T. T.; Keech, J. T.; Peake, G. T.; Santarsiero, B. D. J. Am. Chem. Soc. 1986, 108, 5333.

[10] Tietze, L. F.; Brand, S.; Pfeiffer, T. Angew. Chem., Int. Ed. 1985, $24,784$.

[11] (a) Hellberg, L. H.; Beeson, C.; Somannathan, R. Tetrahedron Lett. 1986, 3955

(b) Sakai, K.; Oisaki, K.; Kanai, M. Adv. Synth. Catal. 2019

(c) Qi, J.; Sun, C.; Tian, Y.; Wang, X.; Li, G.; Xiao, Q.; Yin, D. Org. Lett. 2014, 16, 190.

(d) Yao, Y.; Li, W.-D.; Tong, W.-T.; Chen, J.-X. Chin. J. Org. Chem. 2015, 35, 223 (in Chinese).

(姚远，李伟东，全文婷，陈建新，有机化学, 2015, 35, 223.)

(e) Chen, C.; Xu, S.-S.; Liu, W.-B. Chin. J. Org. Chem. 2016, 36, 1890 (in Chinese).

(陈翠, 徐松森, 刘卫兵, 有机化学, 2016, 36, 1890.)

[12] (a) Gresham, T. L.; Jansen, J. E.; Shaver, F. W.; Bankert, R. A.; Fiedorek, F. T. J. Am. Chem. Soc. 1951, 73, 3168.

(b) Guo, W.; Gómez, J. E.; Martínez-Rodríguez, L.; Bandeira, N. A.; Bo, C.; Kleij, A. W. ChemSusChem 2017, 10, 1969.

[13] (a) Matsumoto, K.; Hashimoto, S.; Okamoto, T.; Otani, S.; Hayami, J. I. Chem. Lett. 1987, 16, 803.

(b) Matsumoto, K.; Hashimoto, S.; Uchida, T.; Okamoto, T.; Otani, S. B. Chem. Soc. Jpn. 1989, 62, 3138.

[14] Chalid, M.; Heeres, H. J.; Broekhuis, A. A. J. Appl. Polym. Sci. 
2012, $123,3556$.

[15] (a) Das, S.; Addis, D.; Knöpke, L. R.; Bentrup, U.; Junge, K.; Brückner, A.; Beller, M. Angew. Chem., Int. Ed. 2011, 50, 9180.

(b) Lei, A.; Waldkirch, J. P.; He, M.; Zhang, X. Angew. Chem., Int. Ed. 2002, 41,4526

(c) Du, X. L.; He, L.; Zhao, S.; Liu, Y. M.; Cao, Y.; He, H. Y.; Fan, K. N. Angew. Chem., Int. Ed. 2011, 123, 7961.

(d) Gao, G.; Sun, P.; Li, Y.; Wang, F.; Zhao, Z.; Qin, Y.; Li, F. ACS Catal. 2017, 7, 4927.

[16] (a) Huang, Y.-B.; Dai, J.-J.; Deng, X.-J.; Qu, Y.-C.; Guo, Q.-X.; Fu, Y. ChemSusChem 2011, 4, 1578.

(b) Touchy, A. S.; Hakim Siddiki, S. M. A.; Kon, K.; Shimizu, K.-I. ACS Catal. 2014, 4, 3045.

(c) Vidal, J. D.; Climent, M. J.; Concepcion, P.; Corma, A.; Iborra, S.; Sabater, M. J. ACS Catal. 2015, 5, 5812.

(d) Wei, Y.; Wang, C.; Jiang, X.; Xue, D.; Li, J.; Xiao, J. Chem.Commun. 2013, 49, 5408.

(e) Sun, Z.; Chen, J.; Tu, T. Green Chem. 2017, 19, 789.

(f) Ledoux, A.; Sandjong Kuigwa, L.; Framery, E.; Andrioletti, B. Green Chem. 2015, 17, 3251.

(g) Du, X.-L.; He, L.; Zhao, S.; Liu, Y.-M.; Cao, Y.; He, H.-Y.; Fan, K.-N. Angew. Chem., Int. Ed. 2011, 50, 7815.

(h) Ogiwara, Y.; Uchiyama, T.; Sakai, N. Angew. Chem., Int. Ed. 2016, 55, 1864

[17] (a) Mansoor, S. S.; Aswin, K.; Logaiya, K.; Sudhan, S. P. N. J. Saudi Chem. Soc. 2016, 20, 138.

(b) Singh, R.; Jakhar, K.; Sharma, P. Chem. Sci. 2017, 6, 135.

(c) Han, L.; Zhou, Z. Appl. Organomet. Chem. 2019, 33, e4755.

[18] Shi, M. S.; Cui, C.; Yin, W. P. Eur. J. Org. Chem. 2005, 11, 2379.

[19] Ghosh, R.; Maiti, S.; Chakraborty, A. Tetrahedron Lett. 2005, 46, 147.
[20] Sun, H. B.; Hua, R.M.; Yin, Y. W. Molecules 2006, 11, 263.

[21] Firouzabadi, H.; Iranpoor, N.; Jafarpour, M.; Ghaderi, A. J. Mol. Catal., A: Chem. 2006, 252, 150

[22] Eftekhari-Sis, B.; Abdollahifar, A.; Hashemi, M. M.; Zirak, M. Eur. J. Org. Chem. 2006, 22, 5152.

[23] Zhang, Z. H.; Li, T. S.; Li, J. J. Catal. Commun. 2007, 8, 1615.

[24] Bhagat, S.; Chakraborti, A. K. J. Org. Chem. 2008, 73, 6029.

[25] Shen, W. L.; Wang, M.; Feng, J. J.; Tian, H. Tetrahedron Lett. 2008, 49, 4047.

[26] Gliozzi, G.; Innorta, A.; Mancini, A.; Bortolo, R.; Perego, C.; Ricci, M.; Cavani, F. Appl. Catal. B-Environ. 2014, 145, 24

[27] Liao, Y.; Liu, Q.; Wang, T.; Long, J.; Ma, L.; Zhang, Q. Green Chem. 2014, 16, 3305.

[28] Li, F.; France, L. J.; Cai, Z.; Li, Y.; Liu, S.; Lou, H.; Li, X. Appl. Catal. B-Environ. 2017, 214, 67.

[29] Antonetti, C.; Melloni, M.; Licursi, D.; Fulignati, S.; Ribechini, E.; Rivas, S.; Galletti, A. M. R. Appl. Catal. B-Environ. 2017, 206, 364.

[30] Wu, C.; Luo, X.; Zhang, H.; Liu, X.; Ji, G.; Liu, Z.; Liu, Z. Green Chem. 2017, 19, 3525 .

[31] (a) Burba, C.; Volland, H. G. US 4156779, 1979.

(b) Nelson, S. G.; Spencer, K. L.; Cheung, W. S.; Mamie, S. J. Tetrahedron 2002, 58, 7081 .

[32] Vollema, G.; Arens, J. F. Recl. Trav. Chim. Pays-Bas 1963, 82, 305.

[33] De Jonge A. P.; Van der Ven B. Recl. Trav. Chim. Pays-Bas 1965, 84, 1177.

[34] Xu, Z.; Yan, P.; Jiang H.; Liu, K.; Zhang, Z. C. Chin. J. Chem. 2017, 35, 581.

[35] Lukeš, R.; Koblicova, Z.; Blaha, K. Chem. Commun. 1963, 28, 2182. 